\title{
Tunable $Q$ matching networks for capacitive ultrasound transmitters
}

\author{
Mansoor Khan ${ }^{1}$ (D) Talha Masood Khan ${ }^{2}$
}

Received: 25 January 2021 / Revised: 25 January 2021 / Accepted: 22 April 2021

(C) The Author(s), under exclusive licence to Springer Science+Business Media, LLC, part of Springer Nature 2021

\begin{abstract}
Airborne capacitive micromachined ultrasonic transducers (CMUTs) have predominantly large input capacitive reactance with small series radiation resistance. To maximize the acoustic power radiation at resonance we employ low cost $L C$ matching networks between the low output impedance driving source and CMUT transducer. Conventional $L C$ networks, including $p i$ and $T$-network topologies are employed to provide a high- $Q$ match. An important free parameter that controls the bandwidth of match in $p i$ and $T$-networks is the center impedance. Since a simple pi-network can be formed from two basic $L$-sections, the center impedance of two $L$-sections provides control over the bandwidth of match. Hence these are tunable- $Q$ matching networks. We use the lumped circuit model of CMUT to derive the input impedance of CMUT at resonance. At resonance the lumped equivalent circuit of transducer can be reduced to a series $R C$ circuit consisting of radiation resistance and transducer's equivalent capacitance only. From the input impedance of CMUT we determine the lumped inductance and capacitances of various $L C$ matching networks. The aim of these matching networks is to cancel the large input capacitive reactance of CMUT cell and to match the cell's radiation resistance to source resistance at resonance. We report significant improvement in the radiated power when CMUT is driven at resonance using the proposed matching schemes.
\end{abstract}

Keywords Impedance matching networks $\cdot$ CMUT $\cdot$ Match bandwidth $\cdot p i$-networks $\cdot T$-networks

\section{Introduction}

A significant advantage of CMUT technology over ceramic transducers is its ease of integration with the front end electronics. Owing to standard micromachining process, fabrication of CMUTs cells in large arrays of arbitrary shape and sizes is much easier compared to piezo transducers. As an ultrasound transmitter, CMUTs has a lot of benefits to offer in applications like high-intensity focused ultrasound [1], intravascular ultrasound [2], medical imaging [3, 4], and non-destructive evaluation [5].

However, the potential of CMUTs as an ultrasound transmitter for therapeutic applications like in tumor ablation using electrical impedance matching networks is

Mansoor Khan

mansoor_khan@comsats.edu.pk

1 Department of Electrical and Computer Engineering, COMSATS University Islamabad, Islamabad 44000, Pakistan

2 Institute of Materials Science and Nanotechnology, Bilkent University, 06800 Ankara, Turkey not much explored. In this paper we use low cost conventional $L C$ matching networks with the signal source to improve the transmitted pressure at resonance. CMUT input impedance is significantly capacitive. This large capacitive reactance when driven by a low source impedance results in lower acoustic energy dissipation. In this paper we show that by using various impedance matching networks this undesired reactance can be eliminated at resonance to permit more real power delivery as in $[6,7]$. By using $L C$ matching networks, like $p i$ and $T$-networks, between the excitation source and CMUT cell a narrowband and a higher level of transmit pressure is achieved. Since both $p i$ and $T$-networks are constructed from two basic $L$-sections, choosing the center impedance of these networks narrows down the bandwidth of match [8]. Hence the center impedance of $p i$ and $T$-networks controls the overall response of match while still mitigating the large undesired capacitive reactance of CMUTs at resonance.

In this work we use the lumped circuit model of CMUT to derive its input impedance at resonance. The lumped circuit elements for $L C$ matching are then determined to match the real part of transducer impedance with source 
resistance while cancelling the large capacitive reactive part of CMUT impedance. Acoustic power radiated into the medium with and without matching networks is obtained from the harmonic balance simulations of large signal model of CMUT. The improvement in radiated power and match bandwidths for various matching networks are then compared.

\section{Impedance matching}

Matching normally means the use of a lossless (nonresistive) network between a signal source and a load in order to maximize the power transferred to the load. Consider an ideal DC voltage generator, $V_{S}$ with its series internal resistance of $R_{S}$ feeding a load resistance of $R_{L}$ as shown in Fig. 1.

For an unequal load and source resistances $R_{L} \neq R_{S}$, power delivered to the load is:

$$
\begin{array}{r}
V_{\text {load }}=\frac{R_{L}}{R_{L}+R_{S}} V_{S} \\
P_{\text {load }}=\frac{V_{\text {load }}^{2}}{R_{L}}=\frac{R_{L}}{\left(R_{L}+R_{S}\right)^{2}} V_{S}^{2}
\end{array}
$$

where $V_{\text {load }}$ is the DC load voltage. Maximum power is transferred when the load resistance is made equal to the source resistance. This can be shown by differentiating the expression for the power in (1) as,

$$
\frac{\partial P_{\text {load }}}{\partial R_{L}}=\frac{V_{S}^{2}}{\left(R_{L}+R_{S}\right)^{2}}-\frac{2 R_{L}}{\left(R_{L}+R_{S}\right)^{3}} V_{S}^{2}=0
$$

Solving for load resistance we obtain from (2), $R_{L}=R_{S}$. Hence the maximum delivered power becomes:

$P_{\text {load }}=\left.\frac{V_{\text {load }}^{2}}{R_{L}}\right|_{R_{L}=R_{S}}=\frac{V_{S}^{2}}{4 R_{L}}$

since half the supply voltage, $V_{S}$ is availabe at the load under maximum power transfer conditions, i.e when $R_{L}=R_{S}$.

\subsection{L-section matching network}

Consider a 2-element $L$-matching network placed between source and load resistance of Fig. 1 Note that the $L$ -

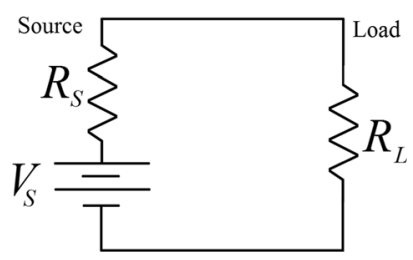

Fig. 1 DC generator driving a resistive load, $R_{L}$ matching network is purely reactive (non-resistive) consisting of two reactive elements only. This configuration is shown in Fig. 2.

Where, $X_{p}$ and $X_{s}$ are the shunt and series reactances respectively. In designing of a $L$-matching network the usual practice is to first place shunt reactive element across the larger resistance and series reactance comes with the smaller resistance $[8,9]$. As an example consider a source resistance, $R_{S}$ of $1000 \Omega$ which is to be matched to a load resistance, $R_{L}$ of $10 \Omega$.

Impedance of the left-hand side, $Z_{\text {left }}$ of circuit in Fig. 2 is given by,

$$
\begin{array}{r}
Z_{\text {left }}=R_{\text {left }}+j X_{\text {left }}=\frac{1000 j X_{p}}{1000+j X_{p}} \\
=\frac{1000^{2} j X_{p}+1000 X_{p}^{2}}{1000^{2}+X_{p}^{2}}
\end{array}
$$

We can pick the value of $X_{P}$ so that the real part of $Z_{\text {left }}$ will be $10 \Omega$, i.e., equal to the load resistance.

That is,

$\operatorname{Re}\left\{Z_{\text {left }}\right\}=R_{\text {left }}=\frac{1000 X_{p}^{2}}{1000^{2}+X_{p}^{2}}=10$

Solving the quadratic in $X_{p}$ of (5) yields $X_{p}= \pm 100.5$, implying inductive reactance $X_{p}=100.5$ or capacitive reactance $X_{p}=-100.5$

The left hand side of circuit now has the correct equivalent series resistance of $10 \Omega$, but it is accompanied by an equivalent series reactance, $X_{\text {left }}$, given by the imaginary part of $Z_{\text {left }}$ in (4). We can cancel this $X_{\text {left }}$ by inserting a series reactor, $X_{S}$, equal to $-X_{\text {left. }}$. That is,

$$
\operatorname{Im}\left\{Z_{\text {left }}\right\}=X_{\text {left }}=\frac{1000^{2} X_{p}}{1000^{2}+X_{p}^{2}}=-X_{S}
$$

where $X_{S}=-99.5$ The final step is to find the values of lumped reactive elements, inductance and capacitances, $L$ and $C$ that produce the specified reactances at the given frequency. Suppose the design frequency is $1.5 \mathrm{MHz}$ $\left(\omega=2 \pi \cdot 1.5 \cdot 10^{6}\right)$.

Then $L=10.66 \mu \mathrm{H}$ and $C=1.07 \mathrm{nF}$. Note that the values of the two reactors are completely determined by the source and load resistances. Except for the choice of which element is to be an inductor and which is to be a capacitor, there are no free parameters in this two-element matching circuit.

Figure 3 shows the power response of circuit with $L$ matching section in Fig. 2 The power response is evaluated by carrying out linear AC analysis of circuit in Advanced Design Systems (ADS) ${ }^{1}$ over a frequency range of up to 5

\footnotetext{
${ }^{1}$ Linear AC analysis of circuit of Fig. 2 is carried out in Advanced Design Systems, Keysight Technologies, Santa Rosa, CA.
} 


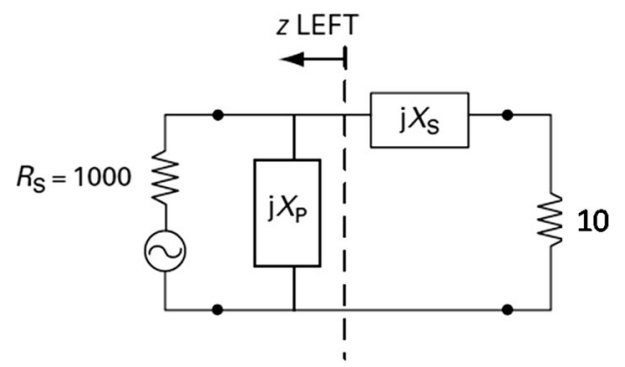

Fig. $2 L$-matching section between source and load resistance

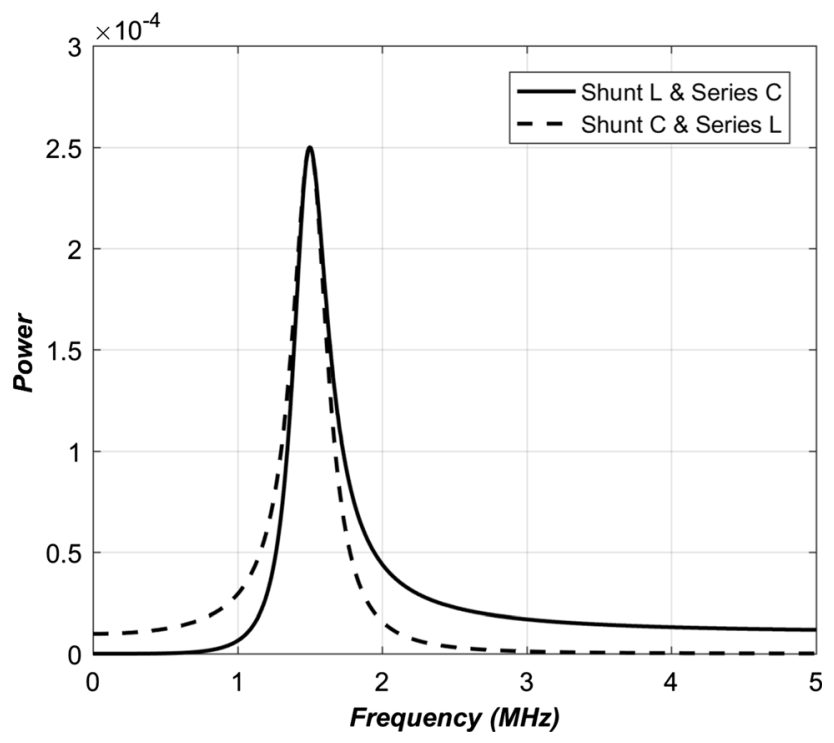

Fig. 3 Power reponse of circuit with $L$-matching section

MHz. Note that the obtained power response depends on the choice of series and shunt reactive elements.

The solid curve of Fig. 3 has shunt inductance and series capacitance, hence no power is delivered to the load at low frequencies around DC. At high frequencies the circuit of Fig. 2 can be redrawn without placing the $L$-matching section between the source and load, thus producing a finite but lower mismatched power output at high frequencies.

Similarly the broken-line curve represents shunt capacitance and series inductance producing zero power output at high frequencies due to shortning of shunt capacitor and opening of series inductor. However a finite but mismatched power is obtained at low frequencies where the shunt capacitor is open and series inductance is a short circuit.

\subsection{Quick design procedure for L-networks}

A well known method for designing of $L$-matching networks is based on quality factor or $Q$ of circuit. Since the values of the two reactors are completely determined by the source and load resistances, its worth mentioning the $Q$ of circuit in terms of load and source resistances depending on which is higher the expression for quality factor can be generalized as:

$$
Q=\sqrt{\frac{R_{\text {high }}}{R_{\text {low }}}-1}
$$

The series and shunt reactances can be found immediately from the $Q$ of (7) as follows:

$$
Q=\frac{R_{\text {high }}}{X_{p}}=\frac{X_{s}}{R_{\text {low }}}
$$

When the ratio of the source resistance to the load resistance is large, the $L$-network produces a narrowband match, i.e., the match will be good only very close to the design frequency (high $Q$ match). Conversely, when the impedance ratio is close to unity, the match is wide (low $Q$ match). Table 1 illustrates the $Q$ of match for varying load resistance, $R_{L}$.

Figure 4 shows the bandwidth of match for varying load resistance, $R_{L}$. As the raio of source to load resistance becomes close to unity the quality factor of match drops and we obtain a wideband match. For higher source to load ratios the match is narrowband (high $Q$ ) at design frequency only.

\section{Tunable $\mathbf{Q}$ matching networks}

\subsection{Pi-matching network}

Higher $Q$ can be obtained with two back-to-back $L$-networks, each one transforming down to a center impedance that is lower than either the generator or the source resistance. The center impedance is a free design parameter that controls the bandwidth of match and hence its quality factor. The resulting $p i$-network is shown below:where $L=$ $L_{1}+L_{2}$ in equivalent $p i$-network topology.

Since the shunt reactive element is placed across the higher impedance, the center impedance, $Z_{\text {center }}$ of both $L$ networks must transform to a lower center impedance than either the source and load impedance, i.e. $Z_{\text {center }}<\min \left\{R_{S}, R_{L}\right\}$. Thus $Z_{\text {center }}$ of pi-network in Fig. 5 is

Table 1 Quality factor of match at $1.5 \mathrm{MHz}$ for varying load resistance, $R_{L}$

\begin{tabular}{lllll}
\hline$R_{L}$ & $Q$ & $X_{P}$ & $X_{S}$ & $R_{S}$ \\
\hline 10 & 9.95 & 100.5 & -99.5 & 1000 \\
100 & 3 & 333.33 & -300 & 1000 \\
500 & 1 & 1000 & -500 & 1000 \\
\hline
\end{tabular}




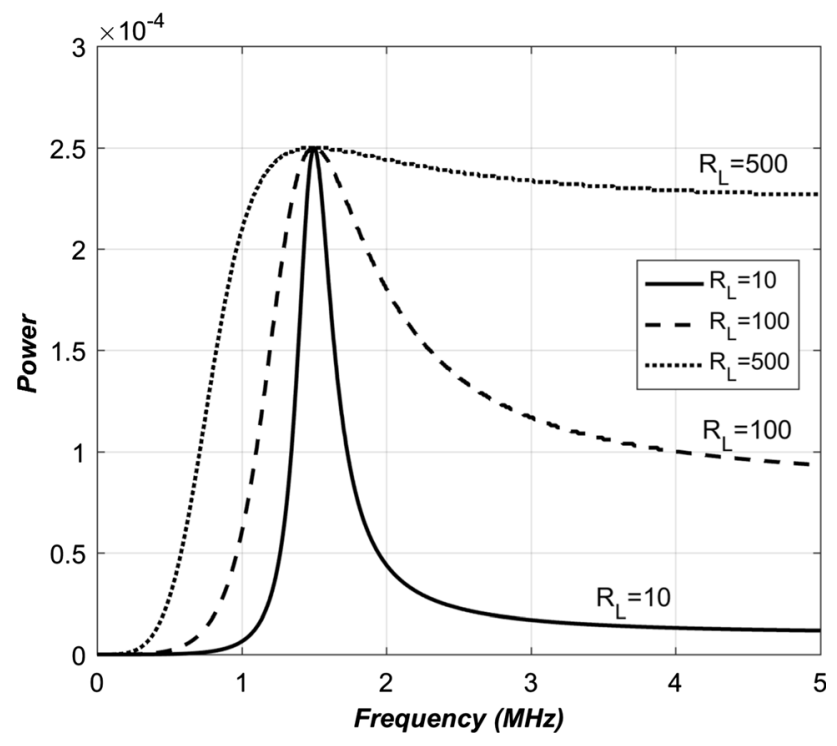

Fig. 4 Power reponse of circuit for varying load resistance, $R_{L}$

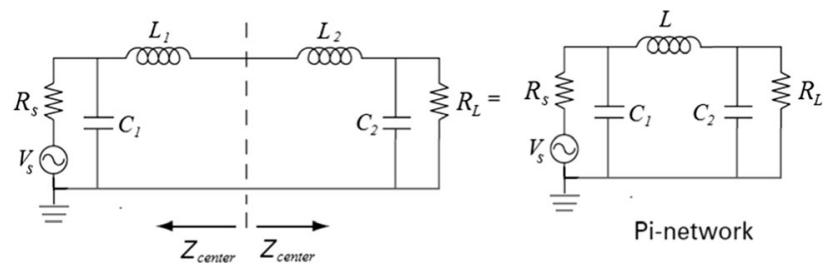

Fig. 5 pi-Network constructed from two back-to-back $L$-networks provides tunable $Q$

a free parameter responsible for the tuning of match bandwidth at the design frequency.

Table 2 gives the lumped element values of $p i$-network obtained from the design equations of (7) and (8) to match a $1000 \Omega$ source to a $50 \Omega$ load at $1.5 \mathrm{MHz}$ design frequency with variable $Z_{\text {center }}$. Figure 6 plots high $Q$ power response at $1.5 \mathrm{MHz}$ for varying $Z_{\text {center }}$.

\subsection{T-matching network}

Combining two "front-to-front" $L$-networks produces the $T$-network as shown in Fig. 7 Each $L$-network transforms

Table 2 Lumped element values of Pi-network at $1.5 \mathrm{MHz}$ for varying center impedance, $Z_{\text {center }}$

\begin{tabular}{llll}
\hline$Z_{\text {center }}(\Omega)$ & $L(\mu H)$ & $C_{1}(n F)$ & $C_{2}(n F)$ \\
\hline 10 & 12.68 & 1.06 & 4.24 \\
20 & 17.45 & 0.74 & 2.59 \\
30 & 20.69 & 0.60 & 1.73 \\
40 & 22.91 & 0.52 & 1.06 \\
\hline
\end{tabular}

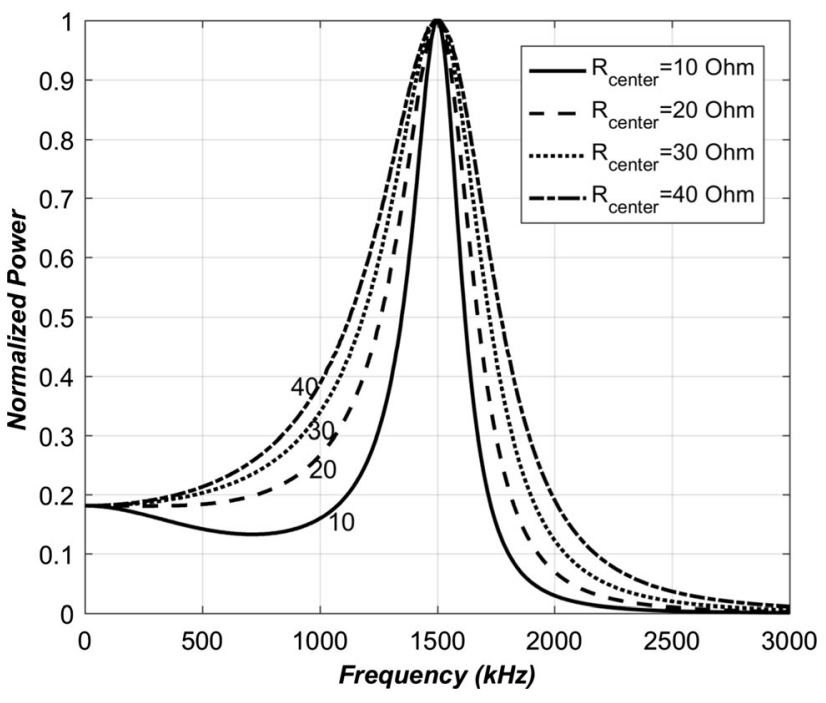

Fig. 6 Variable $Q$ pi-network power response

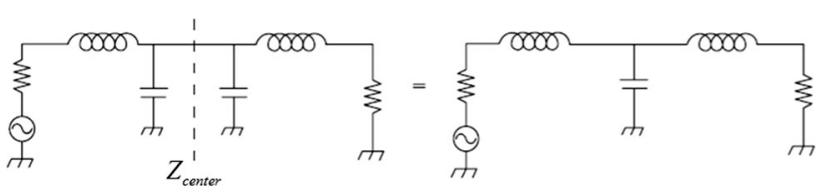

Fig. 7 -matching network

up to a center impedance that is higher than both the source and load impedances. Note that both the pi-network and the $T$-network have a free parameter (the center impedance) which gives us some control over the frequency response while still providing a perfect match at the center or design frequency.

Figure 8 plots the power response of $T$-network used to match a $1000 \Omega$ source with a $50 \Omega$ load. The center

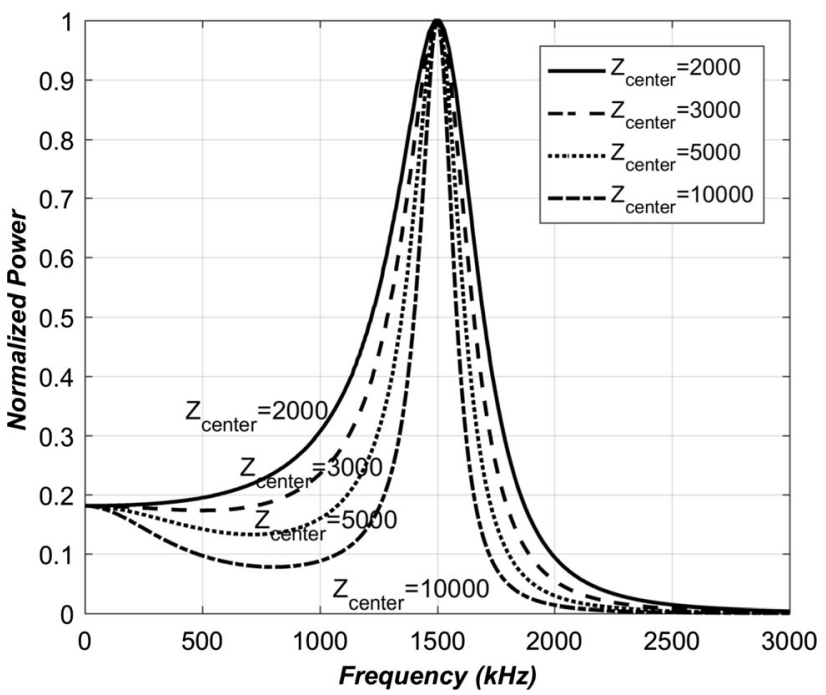

Fig. $8 T$-network power response for varying center impedance, $Z_{\text {center }}$ 
impedance $Z_{\text {center }}$ is varied between $2000<Z_{\text {center }}<10,000$ to produce a high $Q$ match at $1.5 \mathrm{MHz}$ design frequency.

\subsection{Lower Q-cascaded L-networks}

In a cascaded double $L$-network (shown in Fig. 9) the first $L$-network transforms to an impedance value between the source and load impedance. The second stage takes it the rest of the way. The process can, of course, be done in smaller steps with any number of cascaded networks. Cascaded $L$-networks produce wider match bandwidth with increasing number of $L$-sections. This is shown in Fig. 10 for a $1000 \Omega$ source to be matched to a $50 \Omega$ load at 1.5 $\mathrm{MHz}$ with multiple $L$-sections.

\section{Lumped equivalent circuit model of a CMUT cell}

Figure 11 shows the basic geomtery of a circular CMUT cell with a suspended (un-collapsed) silicon membrane. Here $a$ is the aperture radius of the CMUT transducer, $t_{m}$ is membrane thickness of top electrode, $t_{i}$ is the insulator thickness $\left(\mathrm{Al}_{2} \mathrm{O}_{3}\right)$ and $t_{g}$ is the physical gap height. $F$ is the overall electric and ambient force being exerted on the membrane. An acoustic wave is generated by applying an electrical signal between the top and bottom electrodes.

The lumped equivalent model of CMUT derived in [10] is obtained by considering the displacement profile $x(r)$ of thin clamped membranes. When depressed by uniform pressure this profile can be expressed as [11],

$x(r)=x_{p}\left(1-\frac{r^{2}}{a^{2}}\right)^{2}$ for $r \leq a$
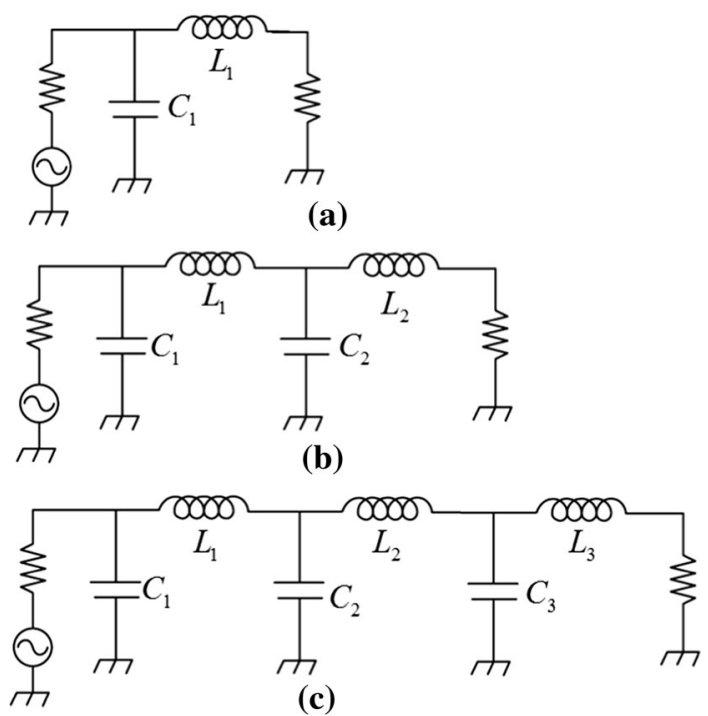

Fig. 9 a Single $L$-section, b double $L$-section, c triple $L$-section

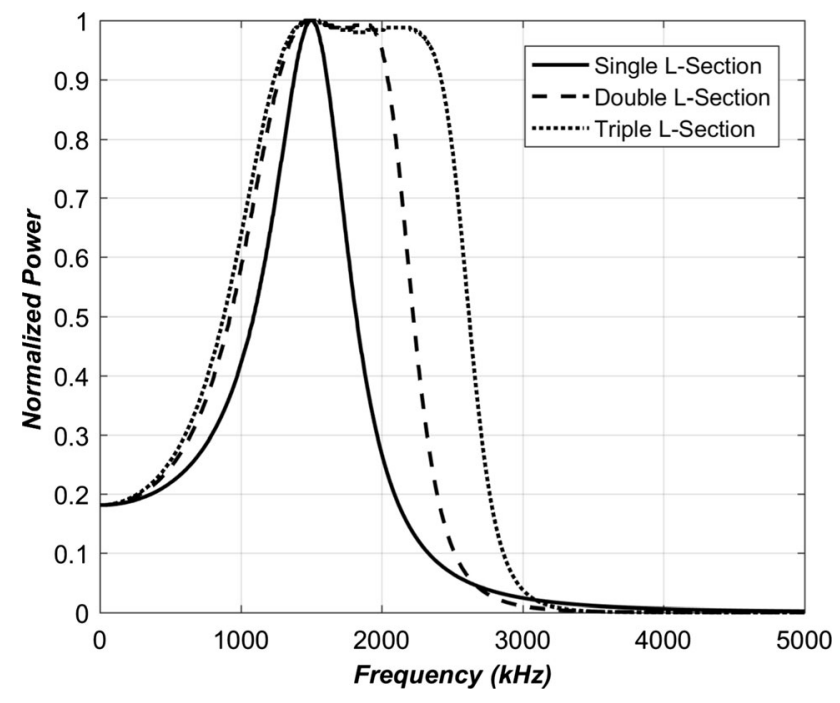

Fig. 10 Power response comparison of multiple $L$-sections

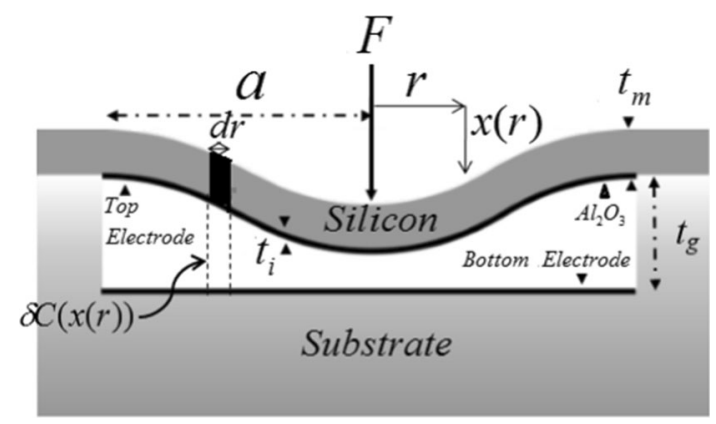

Fig. 11 Cross-sectional view of a circular CMUT cell

where $r$ is the radial membrane position and $x_{p}$ is the peak displacement at the membrane center.

The incremental capacitance, $\delta C(r)$, of a concentric narrow ring on the membrane of radius $r$ and width $d r$ can be expressed as:

$$
\delta C(r)=\frac{\epsilon_{0} 2 \pi r d r}{t_{g e}-x(r)}=\frac{\epsilon_{0} 2 \pi r d r}{t_{g e}-x_{p}\left(1-\frac{r^{2}}{a^{2}}\right)^{2}}
$$

where $t_{g e}=t_{g}+t_{i} / \epsilon_{r}$ is the effective electrical height of CMUT gap inclusive of both insulator and gap capacitances in series. $\epsilon_{0}$ and $\epsilon_{r}$ are the electric permittivities of gap and insulator material respectively.

Total capacitance of the deflected membrane of radius $a$ becomes:

$$
C=\int_{0}^{a} \delta C(r) d r=C_{0} g\left(\frac{x_{p}}{t_{g e}}\right)
$$

where the function $g\left(\frac{x_{p}}{t_{g e}}\right)=\tanh ^{-1}\left(\sqrt{\frac{x_{p}}{t_{g e}}}\right) / \sqrt{\frac{x_{p}}{t_{g e}}}$ and $C_{0}=\epsilon_{0} \pi a^{2} / t_{g e}$.

If an ac signal $V(t)$ is applied between the electrodes of the transducer, the instantaneous energy stored in the 
capacitance becomes: $E(t)=1 / 2 C(t) V^{2}(t)$. The instantaneous spatial rms membrane velocity described in [12] as spatial rms velocity is the through variable of equivalent circuit given by:

$$
v_{R}(t)=\frac{d x_{R}(t)}{d t}=\frac{d}{d t} \sqrt{\frac{1}{\pi a^{2}} \int_{0}^{a} 2 \pi r x^{2}(r, t) d r}
$$

where $x_{R}(t)=x_{P}(t) / \sqrt{5}$ for the membrane profile described in (9). To preserve the energy in the model, the corresponding across variable for force, $f_{R}(t)$, is espressed as:

$$
\begin{aligned}
& f_{R}(t)=\frac{\partial E(t)}{\partial x_{R}}=\sqrt{5} \frac{\partial E(t)}{\partial x_{p}} \\
& f_{R}(t)=\sqrt{5} \frac{C_{0} V^{2}(t)}{2 t_{g e}} g^{\prime}\left(\frac{x_{p}}{t_{g e}}\right)
\end{aligned}
$$

Electrical side of lumped equivalent circuit is found by considering the time rate of change of the instantaneous charge, $Q(t)=C(t) V(t)$, on the CMUT capacitance as:

$$
\frac{\partial Q(t)}{\partial t}=C(t) \frac{\partial V(t)}{\partial t}+V(t) \frac{\partial C(t)}{\partial t}=i_{C a p}(t)+i_{V}(t)
$$

The right handside of (14) are the current components at the electrical side of CMUT circuit shown in Fig. 12 These two current components can be expressed in terms of CMUT instantaneous capacitance, $C(t)$ and force $f_{R}(t)$ as:

$$
\begin{array}{r}
i_{\text {Cap }}(t)=C(t) \frac{d V(t)}{d t}=C_{0} \frac{d V(t)}{d t}+i_{C}(t), \\
\text { where } i_{C}(t)=\left(C(t)-C_{0}\right) \frac{d V(t)}{d t}
\end{array}
$$

The velocity current is given by,

$$
i_{V}(t)=V(t) \frac{\partial C(t)}{\partial t}=\frac{\partial C(t)}{\partial x_{R}} \frac{\partial x_{R}}{\partial t}
$$

Considering (14), and $C(t)=2 E(t) / V^{2}(t)$, the velocity current of (17) can be rewritten in terms of instantaneous rms force on membane as:

$$
i_{V}(t)=\frac{2 f_{R}(t)}{V(t)} v_{R}(t)=\sqrt{5} \frac{C_{0} V(t)}{t_{g e}} g^{\prime}\left(\frac{x_{p}}{t_{g e}}\right) v_{R}(t)
$$

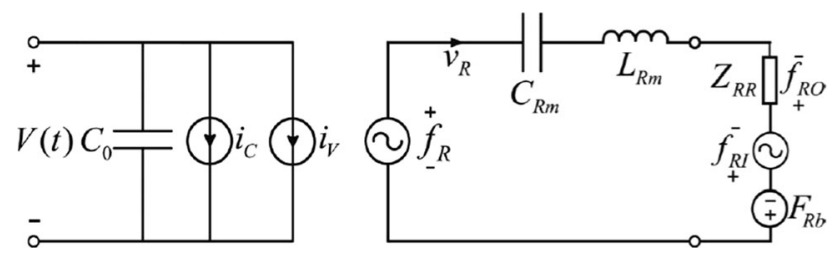

Fig. 12 Lumped equivalent circuit model of CMUT with the rms membrane velocity, $v_{R}$ defined as the through variable and $r m s$ force, $f_{R}$ as the across variable at the mechanical side
$L_{R m}$ is the lumped inductance in the $r m s$ model and is exactly equal to the mass of the membrane.

$$
L_{R m}=\pi a^{2} \rho t_{m}
$$

where $\rho$ is the density of membrane material. $L_{R m}$ interacts with series capacitance, $C_{R m}$ to produce mechanical resonance in the medium. This capacitance represesnts the compliance of membrane. The resonance frequency of membrane depends on both the material and geometrical parameters of CMUT cell. Compliance capacitance $C_{R m}$ is given by,

$$
C_{R m}=\frac{9\left(1-\sigma^{2}\right) a^{2}}{80 \pi Y_{o} t_{m}^{3}}
$$

where $Y_{o}$ and $\sigma$ are the Young's modulus and poisson ratio of plate material.

For the same model, $Z_{R R}$ is the radiation impedance of the CMUT cell. It determines the amount of acoustical power that is generated in the radiation medium given the membrane motion. $Z_{R R}$ of CMUT cell can be calculated by dividing the total acoustic power, $P$, on the surface of the CMUT cell to the square of the absolute value of the spatial rms velocity, $v_{R}$, of the plate as described in [13] and [14]:

$$
Z_{R R}=\frac{P}{\left|v_{R}^{2}\right|}=\frac{\int_{S} p(r) v^{*}(r) d S}{\left|v_{R}^{2}\right|}
$$

where $p(r)$ and $v(r)$ are the pressure and the particle velocity as a function of the radial distance, $r$ and $S=\pi a^{2}$ is the surface area of the CMUT cell. Radiation impedance of CMUT transducer with a certain velocity profile is given in appendix A. $f_{R I}$ and $F_{R b}$ account for incident dynamic acoustic signal and static atmospheric pressure in the model.

\section{Harmonic balance analysis of CMUT's lumped circuit}

We use harmonic balance (HB) to analyze the steady state response of nonlinear lumped circuit model of CMUT of Fig. 12 as in [15]. The input to the system is assumed to be a sinusoid, and the steady-state solution is found as the sum of a fundamental component and its harmonics. HB analysis of the linear mechanical section consisting of lumped elements, $C_{R m}$ and $L_{R m}$ including the radiation impedance of CMUT cell is modelled in Advanced Design Systems (ADS). The radiation impedance, $Z_{R R}$ is defined in frequency domain using symbolically defined device (SDD) of the HB simulator. SDD enables us to create, multiport nonlinear equation-based components. We implemented the physical equations of Sect. 4 for CMUT by relating port currents, port voltages, and their derivatives in this device. 
The transduction force of (14), $f_{R}(t)$ is used to generate the force on the mechanical side of circuit. Equations (16) and (18) gives the capacitor and velocity currents, on the electrical side of the model. Normalized membrane peak displacement, $x_{p} / t_{g e}$ of (11) and (14) is found from the static analysis considering the static force equilibrium. For completeness the calculations of $x_{p} / t_{g e}$ for the given applied DC voltage, $V_{D C}$ between the electrodes in presence of ambient medium loading is presented in Appendix B. As a consequence of the model we can calculate the transmitted power, force and capacitive and velocity currents as outputs for CMUT of arbitrary geomterical dimensions. Table 3 lists the material and dimensional parameters of an airborne CMUT cell which is modelled using the lumped circuit.

First the static membrane displacement of $X_{P} / t_{g e}=0.75$ is found out for the given CMUT operating conditions of normalized force, $F_{b} / F_{g}$ and bias, $V_{D C} / V_{r}$ from Fig. 18 The normalized membrane displacement $X_{P} / t_{g e}$ is then used to define the CMUT capacitance in (11) and force of (14) in the model.

\subsection{Mechanical resonance of CMUT membrane}

As a consequence of model, the mechanical resonance of CMUT is directly obtained from its lumped inductance, $L_{R m}$ and compliance capacitance, $C_{R m}$. For the geometrical parameters and material properties of CMUT cell listed in Table 3, the mechanical resonance of CMUT plate can be calculated as:

$$
f_{r}=\frac{1}{2 \pi \sqrt{L_{R m} C_{R m}}}=\frac{2}{3 \pi} \frac{t_{m}}{a^{2}} \sqrt{\frac{5 Y_{o}}{\rho\left(1-\sigma^{2}\right)}}=77.6 \mathrm{kHz}
$$

Figure 13 shows the input impedance of CMUT circuit model with mechanical resonance of $77.6 \mathrm{kHz}$, at this frequency the series combination of mechanical reactances

Table 3 Dimensional parameters of a CMUT cell

\begin{tabular}{lll}
\hline$a$ & Membrane radius & $1.4 \mathrm{~mm}$ \\
$t_{m}$ & Membrane thickness & $40 \mu \mathrm{m}$ \\
$t_{g e}$ & Effective gap height & $10 \mu \mathrm{m}$ \\
$t_{i}$ & Insulation layer & $100 \mathrm{~nm}$ \\
$V_{D C}$ & DC bias & $20 \mathrm{~V}$ \\
$F_{b} / F_{g}$ & Normalized static force & 0.75 \\
$Y_{0}$ & Young's modulus & $148 \mathrm{GPa}$ \\
$\rho_{m}$ & Si plate density & $2370 \mathrm{~kg} / \mathrm{m}^{3}$ \\
$\sigma$ & Poisson's ratio & 0.17 \\
$\epsilon_{r}$ & Dielectric constant & 9.5 \\
\hline
\end{tabular}

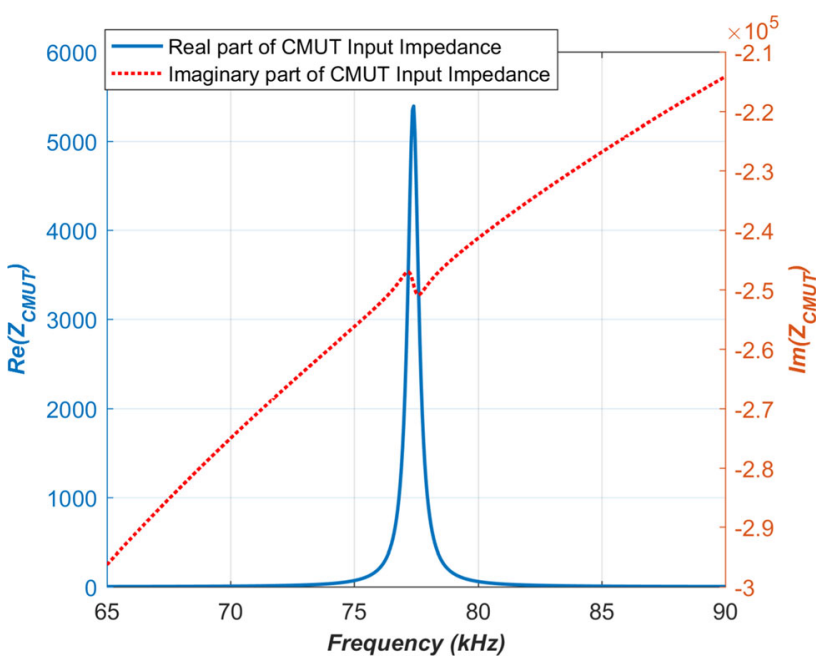

Fig. 13 Simulated CMUT input impedance

$L_{R m}$ and $C_{R m}$ becomes short resulting in a large velocity current, $v_{R}$ through the mechanical part of the circuit of Fig. 12 Hence at resonance the CMUT plate couples maximum real power into the radiating medium. The amount of total acoustic power generated into the medium can be found from the model directly from the plate velocity, $v_{R}$ and the radiation impedance, $Z_{R R}$ as given in eq (21).

\subsection{Input impedance of CMUT cell at resonance}

At resonance, $Z_{C M U T}=5391-j 2.49 \times 10^{5} \Omega$ is predominantly capacitive as can be seen in Fig. 13 Hence the thevenin input impedance of CMUT cell can be represented by a series $R C$ circuit at resonance. Where $C$ is the equivalent CMUT capacitance of $8.24 \mathrm{pF}$ and $R$ is the loss accounting for the radiation resistance of $5391 \Omega$ at resonance.

\section{Electrical impedance matching networks for CMUT cell}

Figure 14 shows various matching networks placed between the source and equivalent series $R C$ circuit of CMUT cell at resonance.

Here $V_{s}$ is an ideal voltage source, with $R_{s}+j X_{s}$ representing the output source impedance. Series $R_{T} C_{T}$ circuit models CMUT transducer load impedance at resonance in all three matching configurations, $X_{1}, X_{2}$ and $X_{3}$ are the reactive components of matching networks, and $Z_{i n}$ is the impedance of the load seen by the source through matching transformation. To match the the output source impedance, $Z_{s}=R_{s}+j X_{s}$ with CMUT load impedance, all three 

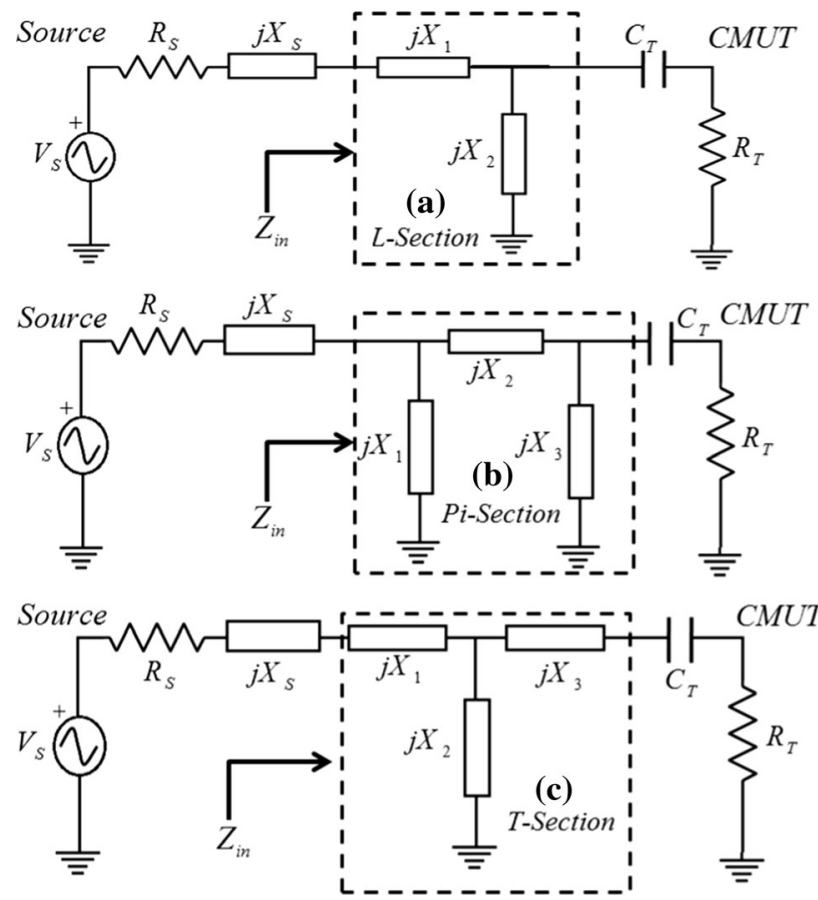

Fig. 14 a $L$-section, b $P i$-section, c $T$-section matching networks for increasing transmitted pressure of CMUT cell at resonance

matching networks must ensure conjugate matching of $Z_{\text {in }}$ with $Z_{s}$, i.e. $Z_{\text {in }}=Z_{s}^{*}$.

\subsection{L-section matching}

For the single $L$-section network of Fig. 14a, we find the reactive elements $X_{1}$ and $X_{2}$ satisfying the conjugate match criteria:

$$
R_{s}=\operatorname{Re}\left\{Z_{\text {in }}\right\} \text { and } X_{s}=-\operatorname{Im}\left\{Z_{\text {in }}\right\}
$$

Satisfying the conditions of (23) equations for $X_{1}$ and $X_{2}$ can be written as in [6]:

$$
\begin{array}{r}
X_{1}=Q R_{s}+X_{s} \text { and } X_{2}=\frac{-\left(R_{T}^{2}-X_{T}^{2}\right)}{Q R_{T}+X_{T}} \\
\text { where } Q= \pm \sqrt{\frac{R_{T}\left[1+\left(\frac{X_{T}}{R_{T}}\right)^{2}\right]}{R_{s}}}
\end{array}
$$

Equation (24) can be simplified if the output source impedance is purely resistive. In this case matching network is a classical $L C$ network. The configuration can be low pass filter or high-pass filter, both configurations are good for matching. However a low-pass filter solution is preferred to avoid the CMUT being excited by any harmonic in the driving source. With these considerations the equivalent circuit of Fig. 14a, will consist of a series inductance, $L_{1}$ in place of reactor $X_{1}$ and a shunt capacitance, $C_{2}$ replacing the reactor $X_{2}$ and the equations in $(24)$ are then reduced to:

$$
\begin{gathered}
\omega L_{1}=Q R_{s}+X_{s} \text { and } \frac{1}{\omega C_{2}}=\frac{-\left(R_{T}^{2}-X_{T}^{2}\right)}{Q R_{T}+X_{T}} \\
\quad \text { where } Q= \pm \sqrt{\frac{R_{T}\left[1+\left(\frac{X_{T}}{R_{T}}\right)^{2}\right]}{R_{s}}}=480
\end{gathered}
$$

A driving source with an output source resistance of $R_{s}=50 \Omega$ is used to drive the CMUT cell at its resonance. To match the transducer impedance, $Z_{C M U T}=R_{T}+j X_{T}=$ $5391-j 2.49 \times 10^{5} \Omega$ with the source impedance, $R_{s}$, we find from (25) the quality of match as, $Q=480$. This yields the match element values as $L_{1}=0.049 H$ and $C_{2}=77.4 p F$. Figure 15 shows the transformed impedance, $Z_{\text {in }}$ of CMUT with $L$-section. As seen by the source, CMUT now appears as a $50 \Omega$ matched load.

\subsection{Pi-matching network}

To maintain the low pass response of matching network, let $X_{1}$ and $X_{3}$ be the shunt capacitances, $C_{1}$ and $C_{3}$ repsectively and $X_{2}$ be a series effective inductance, $L_{2}$ in Fig. 14b. Table 4 lists the values of pi-network $L C$ components for variable matching $Q s$.

\subsection{T-matching network}

For the low pass response of $T$-matching network, let $X_{1}$ and $X_{3}$ be series inductors, $L_{1}$ and $L_{3}$ repsectively and $X_{2}$ be the shunt effective capacitance, $C_{2}$ in Fig. 14c. Table 5 lists the values of $T$-network $L C$ components for variable matching $Q s$.

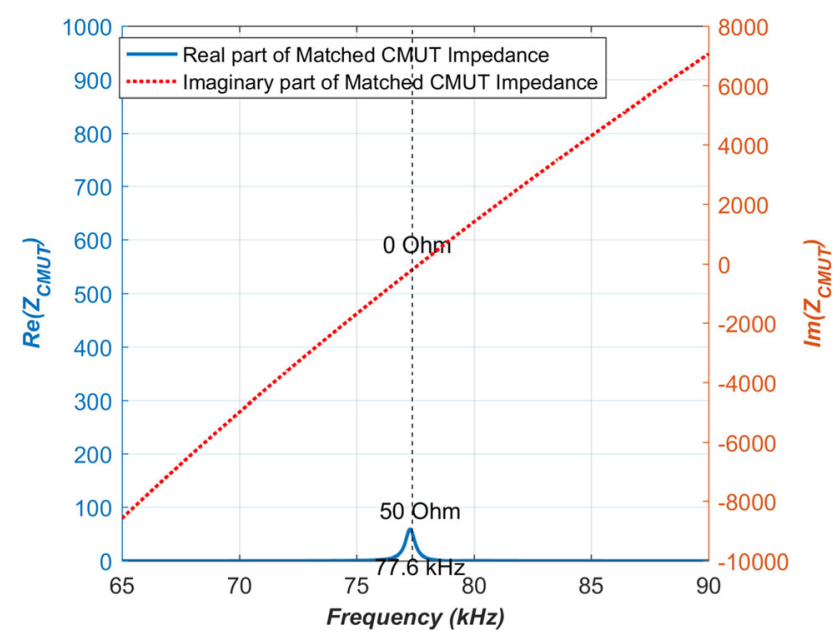

Fig. 15 Transformed impedance of CMUT, $Z_{\text {in }}$ through $L$-section matching network 
Table 4 Lumped element values for matching Pi-network at resonance

\begin{tabular}{llll}
\hline$Q$ & $C_{1}(\mu F)$ & $L_{2}(m H)$ & $C_{3}(n F)$ \\
\hline 300 & 1.18 & 1.71 & 2.46 \\
480 & 1.89 & 1.06 & 3.94 \\
1000 & 3.95 & 0.51 & 8.23 \\
\hline
\end{tabular}

Table 5 Lumped element values for matching T-network at resonance

\begin{tabular}{llrl}
\hline$Q$ & $L_{1}(H)$ & $C_{2}(p F)$ & $L_{3}(H)$ \\
\hline 300 & 0.03 & 149.88 & 0.83 \\
480 & 0.049 & 93.68 & 1.02 \\
1000 & 0.10 & 44.97 & 1.58 \\
\hline
\end{tabular}

\section{Improvement in acoustic power radiated by CMUT cell under matched conditions}

Figure 14 shows CMUT transducer at resonance only as a series $R C$ load. The power after matching section and before $R C$ load is the total electrical power available at the input of transducer. To evaluate the total acoustic power radiated into the medium, we employ large signal model after the matching section as shown in Fig. 16 Radiated power into the medium with matching is calculated from the velocity current, $v_{R}$ and the radiation impedance of the cell, $Z_{R R}$ as: $P=Z_{R R}\left|v_{R}\right|^{2}$. Transducer is driven at resonance frequency only with matching network. Table 6 compares the radiated power output of CMUT with various matching networks with the same $Q$.

As expected matching significantly improves the radiated power in all three cases, however $L$-section matching is the most inexpensive way of achieving higher radiated power with two reactive elements only. If higher match $Q$ or variable match bandwidths are required then atleast three reactive element based matching networks like $p i$ and

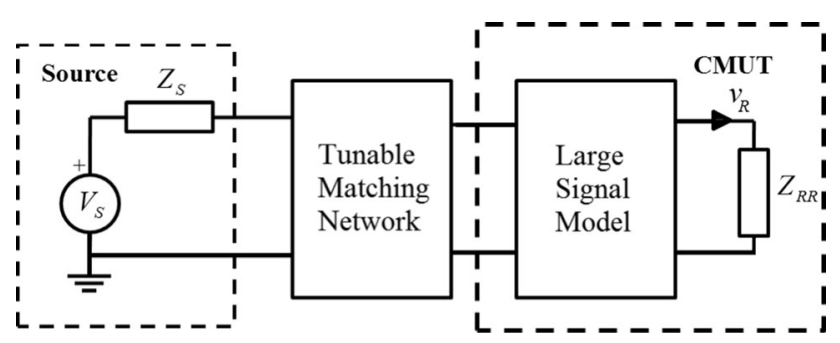

Fig. 16 Evaluation of radiated power under matched conditions using radiation impedance $Z_{R R}$ and particle velocity, $v_{R}$ over the plate surface
$T$ should be utilized. Table 7 compares the transducer radiated output power for different match bandwidths. For the same match $Q$, the radiated power is higher with $T$ networks compared to $p i$ and $L$-section networks.

\section{Conclusion}

This work shows that the transmit peformance of CMUT can be significantly improved if its large capacitive input impedance is matched to the the source output impedance. This can be done by employing conventional $L C$ matching networks, including $p i$ and $T$-network topologies which are capable of producing a tunable match bandwidth owing to their variable center impedances. All three matching toplogies are evaluated with the lumped circuit model simulation of CMUT. The proposed matching schemes enable CMUTs to radiate significantly higher acoustic pressures with better signal-to-noise ratio in immersion mediums.

\section{Appendix A}

\section{Radiation impedance}

For a certain velocity profile, the self radiation impedance of a transducer is found by considering the ratio of total radiated power to the square of the absolute value of a nonzero reference velocity as [16]:

$$
Z_{R R}=\frac{\int_{S} p(r) v^{*}(r) d S}{V_{i} V_{i}^{*}}=\frac{P_{\text {total }}}{\left|V_{i}^{2}\right|}
$$

If the reference velocity, $V_{i}$ is chosed as the rms velocity, the radiation impedance derived in [17] becomes:

$$
Z_{\text {Rrms }}=\pi a^{2} \rho_{0} c\left(1-\frac{20}{(k a)^{9}}\left[F_{1}(2 k a)+j F_{2}(2 k a)\right]\right)
$$

where $\rho_{0}$ is the density and $c$ is the speed of sound in medium and

$$
\begin{array}{r}
F_{1}(y)=\left(y^{4}-91 y^{2}+504\right) J_{1}(y)+14 y\left(y^{2}-18\right) J_{0}(y) \\
-y^{5} / 16-y^{7} / 768
\end{array}
$$

and 
Table 6 Magnitude of acoustic radiated power by CMUT in air at resonance under matched conditions

\begin{tabular}{lllll}
\hline Matching network & $Q$ & Power v & Power without matching & Improvement factor \\
\hline L-Section & 480 & $3.89 \times 10^{-6}$ & $7.3 \times 10^{-8}$ & 53 \\
Pi Network & 480 & $3.48 \times 10^{-6}$ & $7.3 \times 10^{-8}$ & 48 \\
T Network & 480 & $5.5 \times 10^{-6}$ & $7.3 \times 10^{-8}$ & 75 \\
\hline
\end{tabular}

Table 7 Magnitude of acoustic radiated power by CMUT in air at resonance at different match bandwidths

\begin{tabular}{lrlll}
\hline Matching network & \multicolumn{1}{c}{$Q$} & Power (magnitude) & Power without matching & Improvement factor \\
\hline Pi Network & 300 & $2.28 \times 10^{-6}$ & $7.3 \times 10^{-8}$ & 31 \\
T Network & 300 & $5.61 \times 10^{-6}$ & $7.3 \times 10^{-8}$ & 77 \\
Pi Network & 1000 & $1.72 \times 10^{-5}$ & $7.3 \times 10^{-8}$ & 24 \\
T Network & 1000 & $5.64 \times 10^{-6}$ & $7.3 \times 10^{-8}$ & 77 \\
\hline
\end{tabular}

$$
\begin{array}{r}
F_{2}(y)=-\left(y^{4}-91 y^{2}+504\right) H_{1}(y)-14 y\left(y^{2}-18\right) H_{0}(y) \\
+14 y^{4} / 15 \pi-168 y^{2} / \pi
\end{array}
$$

where $J_{n}$ and $H_{n}$ are the $n t h$ order Bessel and Struve functions, respectively. $Z_{R R}$ is plotted in Fig. 17.

Real part of $Z_{R R}$ is the radiation resistance that represents the amount of energy propagated by the CMUT in the medium. The imaginary part of $Z_{R R}$ or the radiation reactance, represents the amount of energy stored in the near vicinity of the transducer in order to facilitate this propagation.

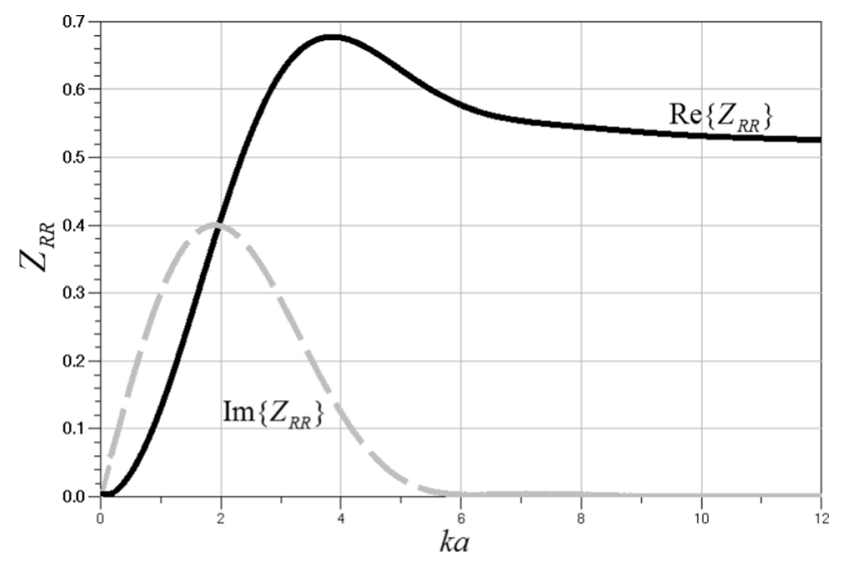

Fig. 17 Radiation Impedance, $Z_{R R}$ of a CMUT cell versus $k a$, where $k$ is the wavenumber in medium of energy propagation

\section{Appendix B}

\section{Static analysis}

In presence of external static pressure, $P_{o}$ due to ambient medium loading, the force $F_{b}=\pi a^{2} P_{o} / 3$ and the electrical force, $F_{p}$ that exists on the membrane due to applied $V_{D C}$ the static peak deflection of membrane $X_{p}$ can be found from the force equilibrium which can be written as $[10,18]$ :

$$
F_{p}+F_{b}=\frac{X_{p}}{5 C_{R m}}
$$

which yields,

$$
\frac{V_{D C}}{V_{r}}=\sqrt{\frac{3\left(\frac{X_{p}}{t_{g e}}-\frac{F_{b}}{F_{g}}\right)}{2 g^{\prime}\left(\frac{X_{p}}{t_{g e}}\right)}} \text { for } \frac{X_{p}}{t_{g e}} \geq \frac{F_{b}}{F_{g}}
$$

where $V_{r}$ is the collapse or pull-in voltage of CMUT in vacuum defined purely in terms of its geometrical and material parameters as:

$$
V_{r}=8 \frac{t_{m}}{a^{2}} t_{g e}^{3 / 2} t_{m}^{1 / 2} \sqrt{\frac{Y_{o}}{27 \epsilon_{0}\left(1-\sigma^{2}\right)}}
$$

and $F_{g}=t_{g e} / 5 C_{R m}$ is the force required to deflect the membrane until the center displacement reaches the gap height, $X_{p}=t_{g e}$. A plot of $X_{p} / t_{g e}$ versus $V_{D C} / V_{r}$ for given operating conditions of $F_{b} / F_{g}$ is plotted in Fig. 18 until collapse threshold.

For given static operating conditions, $V_{D C} / V_{r}$ and $F_{b} / F_{g}$, the corresponding normalized membrane 


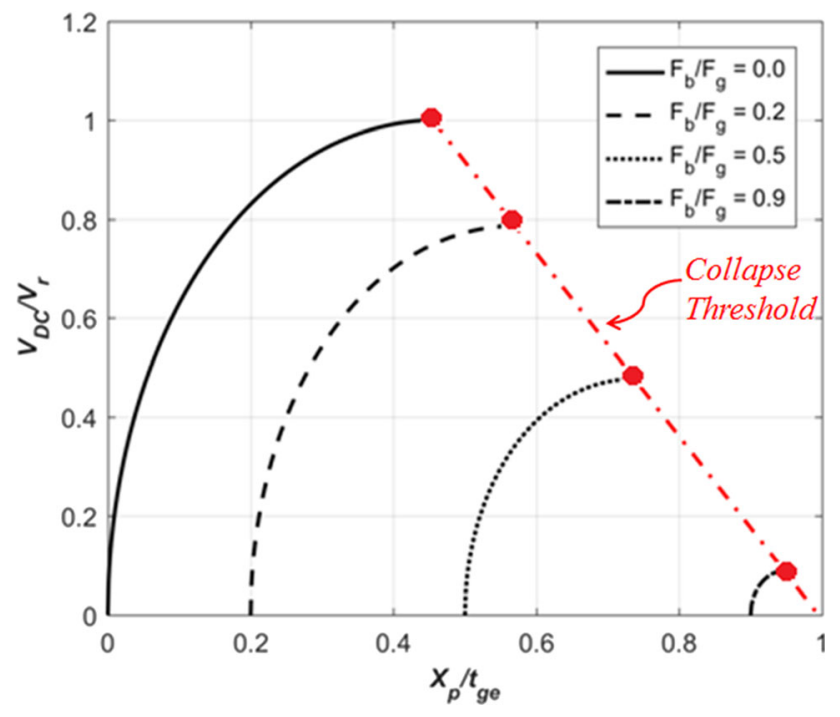

Fig. 18 Normalized peak membrane displacement $X_{p} / t_{g e}$ for given static operating conditions of $V_{D C} / V_{r}$ and normalized force, $F_{b} / F_{g}$ until membrane reaches the substrate and collapse occurs

displacement, $X_{p} / t_{g e}$ is obtained from Fig. 18 and is used in the lumped model to define the CMUT capacitance, force and velocity current. Note that the normalized force $F_{b} / F_{g}$ is completely determined by the CMUTs geometry and material parameters and is given by:

$$
\frac{F_{b}}{F_{g}}=\frac{3 \pi a^{4}\left(1-\sigma^{2}\right) P_{o}}{16 \pi Y_{o} t_{g e} t_{m}^{3}}
$$

\section{References}

1. Wong, S. H., Kupnik, M., Watkins, R. D., Butts-Pauly, K., \& Khuri-Yakub, B. T. (2009). Capacitive micromachined ultrasonic transducers for therapeutic ultrasound applications. IEEE Transactions on Biomedical Engineering, 57(1), 114-123.

2. Degertekin, F. L., Guldiken, R. O., \& Karaman, M. (2006). Annular-ring CMUT arrays for forward-looking IVUS: Transducer characterization and imaging. IEEE Transactions on Ultrasonics, Ferroelectrics, and Frequency Control, 53(2), 474-482.

3. Mills, D. M., Smith, L. S.,\&" Real-time in-vivo imaging with capacitive micromachined ultrasound transducer (CMUT) linear arrays. In Ultrasonics, 2003. IEEE symposium (Vol. 1, pp. 568-571). IEEE, 2003.

4. Mills, D. M. (2004). Medical imaging with capacitive micromachined ultrasound transducer (CMUT) arrays. In Ultrasonics Symposium, 2004. IEEE (Vol. 1, pp. 384-390). IEEE, 2004.

5. Wong, L. L. P. (2014). Capacitive micromachined ultrasonic transducers for non-destructive testing applications. Doctoral dissertation, University of Waterloo.

6. Garcia-Rodriguez, M., Garcia-Alvarez, J., Yanez, Y., GarciaHernandez, M., Salazar, J., Turo, A., et al. (2010). Low cost matching network for ultrasonic transducers. Physics Procedia, 3(1), 1025-1031.
7. Rathod, V. T. (2019). A review of electric impedance matching techniques for piezoelectric sensors, actuators and transducers. Electronics, 8(2), 169.

8. Hagen, J. B. (2009). Radio-frequency electronics: Circuits and applications. Cambridge: Cambridge University Press.

9. Coleman, C. (2004). An introduction to radio frequency engineering. IET.

10. Koymen, H., Atalar, A., Aydogdu, E., Kocabas, C., Oguz, H. K., Olcum, S., et al. (2012). An improved lumped element nonlinear circuit model for a circular CMUT cell. IEEE Transactions on Ultrasonics, Ferroelectrics, and Frequency Control, 59(8), 1791-1799.

11. Timoshenko, S. P., \& Woinowsky-Krieger, S. (1959). Theory of plates and shells. New York: McGraw-Hill.

12. Wallace, C. (1972). Radiation resistance of a baffled beam. The Journal of the Acoustical Society of America, 51(3B), 936-945.

13. Ozgurluk, A., Atalar, A., Koymen, H., \& Olcum, S. (2011). Radiation impedance of an array of circular capacitive micromachined ultrasonic transducers in collapsed state. In IEEE International ultrasonics symposium (pp. 1020-1023). IEEE, 2011.

14. Ozgurluk, A., Atalar, A., Koymen, A., \& Olcum, H. (2012). Radiation impedance of collapsed capacitive micromachined ultrasonic transducers. IEEE Transactions on Ultrasonics, Ferroelectrics, and Frequency Control, 59(6), 1301-1308.

15. Oguz, H. K., Olcum, S., Senlik, M. N., Tas, V., Atalar, A., \& Koymen, H. (2010). Nonlinear modeling of an immersed transmitting capacitive micromachined ultrasonic transducer for harmonic balance analysis. IEEE Transactions on Ultrasonics, Ferroelectrics, and Frequency Control, 57(2), 438-447.

16. Foldy, L. L. (1949). Theory of passive linear electroacoustic transducers with fixed velocity distribution. The Journal of the Acoustical Society of America, 21(6), 595-604.

17. Greenspan, M. (1979). Piston radiator: Some extensions of the theory. The Journal of the Acoustical Society of America, 65(3), $608-621$

18. Köymen, H., Atalar, A., \& Oğuz, H. K. (2012). Designing circular CMUT cells using CMUT biasing chart. In IEEE international ultrasonics symposium (pp. 975-978). IEEE, 2012.

Publisher's Note Springer Nature remains neutral with regard to jurisdictional claims in published maps and institutional affiliations.

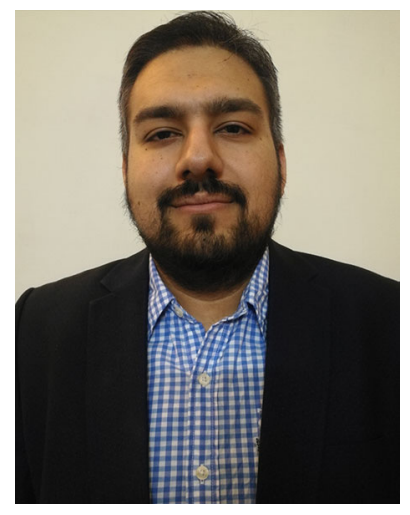

Mansoor Khan received the B.S. degree in electrical and electronics engineering from National University of Sciences and Technology, Karachi, Pakistan, in 2007 and M.Sc. degree in signal processing from Nanyang Technological University, Singapore, in 2009. He received his $\mathrm{Ph} . \mathrm{D}$. degree in electrical and electronics engineering at Bilkent University, Ankara, Turkey in 2018. He is with the Department of Electrical and Computer Engineering at COMSATS University, Islamabad, Pakistan. His research interests include digital filter design, analog filter design, digital signal processing and linear circuit modelling of microelectromechanical systems as sensors. 


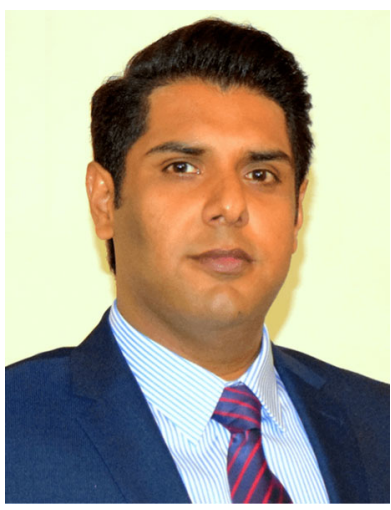

Talha Masood Khan received the M.S. degree in electrical engineering from the National University of Sciences and Technology, Islamabad, Pakistan, in 2013. He received his $\mathrm{Ph} . \mathrm{D}$. degree in material science and nanotechnology from the National Nanotechnology Center, Bilkent University, Ankara, Turkey in 2020. His research interests include Bio-MEMS, MEMS sensors, airborne acoustics, and ultrasonic transducer design. 